\title{
Physical Chemistry and Physiology at the British Association.
}

Interfacial Phenomena.

I $N$ the Physiology Section, at the recent meeting of the British Association at Liverpool, important communications on this subject were given by Prof. W. Ramsden and collaborators, and some remarkably pretty demonstrations were shown.

Mr. J. R. Bruce and Prof. W. Ramsden showed that egg-albumin became irreversibly coagulated at the gas-water surface, even when all such mechanical disturbances as could compress the adsorbed protein film laterally were strictly excluded. The solubility or insolubility of the adsorptum was ascertained in situ by subjecting the rigid adsorption surface to three different treatments:- $(\mathrm{I})$ it was washed from below with large volumes of water ; (2) bile salt was introduced into the depths of the underlying solution ; (3) it was made continuous with a surrounding surface of water maintained in a clean condition and of full normal surface-tension. If the surface rigidity persisted, it was argued that the adsorbed protein had lost its initial solubility. It was concluded that with egg-albumin coagulation took place by the catalytic influence of surface-conditions, and that gross mechanical factors played no essential part,"mechanical surface coagula" should be termed " massed surface coagula.."

True coagulation was a dehydrating condensation of the amino and carboxyl groups of large numbers of neighbouring protein molecules. Metaprotein formation was a precisely similar condensation of a relatively small number of molecules. The size of the complexes formed depended mainly on the concentration of the protein at the time when the reacting groups were activated. Protein adsorbed at a gas/water interface was highly concentrated and the denaturation which followed resulted therefore in the production of coagulated protein. It was also shown (by method 3) that egg-albumin, fibrinogen and edestin became irreversibly coagulated within less than five seconds of attaining a gas-water surface.

Mr. J. Brooks and Prof. W. Ramsden showed that interfaces between water and benzene or water and paraffin in the presence of various emulsifying soluble solids were in some cases mobile, in others rigid. The existence of such mobility showed that Bancroft's theory that stabilisation of emulsions was effected by a continuous emulsifying shell with two different surface tensions on its two faces was in need of important modification.

In cases where the emulsifying substance consisted of insoluble solids in fine suspension, evidence was given that the chief factor determining which of the two liquids became dispersed in the other was the angle of contact formed between the liquid-liquid interfaces and the sides of discrete solid particles. Methods were given for ascertaining in which of the two liquids the angle of contact was obtuse, and it was found that in every case it was this liquid which became dispersed in the other.

The demonstrations, given by Prof. W. Ramsden and Miss A. Mackenzie, to illustrate experiments on surface-films, were very beautiful. One simple experiment to illustrate the rigidity of surface-films in certain cases can easily be repeated by any one : a light magnet is floated on the surface of a saponin solution, and an ordinary pivoted magnetic needle immersed in the same solution. On bringing a magnet near to the vessel, the surface magnet remains stationary, while the immersed one follows the movements of the magnet outside just as readily as it would do in air.
As Prof. Donnan pointed out in his presidential address to the Section of Chemistry, many substances spread on water surfaces to a stable film, one molecule thick. All the molecules appear to be oriented parallel to one another and perpendicular to the surface. Mr. N. K. Adam, who has employed this method for the determination of the cross-sectional area of molecules, gave a demonstration at the scientific soirée of the method of procedure. He has been able further to show that these surface films possess, according to the conditions; the properties of solids, liquids, or gases, a fact of the greatest theoretical significance.

\section{The Properties of Membranes.}

A joint discussion on "The Physical Chemistry of Membranes in Relation to Physiological Science "was held by the Chemistry and Physiology Sections, and was opened by Prof. H. E. Roaf. A membrane was defined as a structure separating two phases; it might be semi-permeable or show permeability of varying grade, and the presence of the membrane made it necessary to consider the possibility of the occurrence of filtration, osmosis, electro-endosmosis, and other related phenomena, for the membrane limited diffusion and allowed differences of concentration of solutes on its two sides, giving rise to various osmotic and electrical phenomena. Physiology was largely concerned with the problem of the passage of material across physiological membranes : as example of these the lungs, intestine, kidney, and salivary gland might be taken.

In the lungs, there appeared to be no certain evidence that the membranes which had to be traversed by the gases entering and leaving the blood did anything but slightly hinder diffusion - the state of equilibrium between blood and air was almost attained, and oxygen never reached a higher partial pressure in the blood than in the air, nor did carbon dioxide ever have a greater pressure in the air of the alveoli than in the blood. Diffusion was adequate to explain not only the partial pressures found in blood and air, but also sufficed to account for the total amounts of oxygen and carbon dioxide traversing the membrane under all conditions.

The passage of substances across the membrane of the intestine offered a much more difficult problem, and one towards the solution of which we had made much less progress, for here many facts seemed to be in opposition to the view that mere diffusion was the chief or even an important factor. When the epithelium was removed from the mucosa of the small intestine, for example, absorption of its contents into the blood was slower, not quicker. Again, blood plasma could be absorbed completely from the lumen of the bowel into the blood, in spite of the apparent identity of the contents with the fluid part of the blood. Finally, when absorption took place from the bowel, the oxygen usage of the bowel had been stated to be increased, i.e. more work was being done by it under these conditions.

The kidney and the salivary gland presented equal difficulties; that considerable work was done by the kidney in concentrating those blood constituents which were excreted, was indisputable. Similarly, the salivary glands could not act by any mere filtration, because, apart from the chemical differences between the blood and the saliva, there was the fact that the pressure reached in the salivary ducts when the flow was stopped by occlusion was, as Ludwig showed, much greater than the maximum arterial 
pressure; one theory which had been advanced to explain this was that the secreting alveolus acted as an osmometer, and attracted fluid from the blood, but this theory leaves us still in somewhat of a dilemma. Some cells, such as the red blood corpuscles, appear to have membranes at their surfaces, but others do not; if an amœba be stained with an intra-vitam dye, the dye does not escape into the surrounding water when the surface of the amøba is punctured.

Prof. F. G. Donnan spolke of membranes from the physico-chemical aspect, but exhibited a good deal of sympathy and interest in the biological side of the question, which he described as one of the most important issues concerned with these physicochemical studies. The fact that membranes might be living structures might alter all physico-chemical conceptions, particularly those based on the study of states of thermodynamic equilibrium. Living organisms utilised an environment not in such an equilibrium, were transformers and consumers of free energy, and environmental equilibrium meant nonactivity and eventual death. Energy potentials might run up in one place and down in another, so that interpretations would be difficult. After referring to the thermodynamic aspect of osmotic pressure, Prof. Donnan reviewed some of the theories which had been advanced in explanation of the properties of membranes. The sieve theory, according to which a semi-permeable membrane acted merely as a sieve, was rather discredited: some form of adsorp- tion theory seemed more attractive; for example, if a substance is negatively adsorbed, it will be repelled from the walls of the pore, so that pure solvent alone passes through. As a modification of this we have various views of ionic adsorption which are capable of explaining many facts. The formation of a Helmholtz double layer on the walls of the pore would explain why the mobility of one ion can be reduced more than that of the other. Different concentrations of electrolyte on the two sides, or a different mobility of ions, would cause a flow by producing electro-endosmosis. The alteration effected in liquidliquid potentials when a membrane was interposed might also be explained on similar lines. (A separate paper contributed to the discussion by Dr. E. B. R. Prideaux also dealt with membrane potentials considered as diffusion potentials.) The product of the activities of two ions on either side of a membrane permeable to both were the same-this is the explanation of the facts of membrane equilibrium (Donnan equilibrium), and is of considerable importance to physiology. The explanation of differential permeability, as given by Meyer and Overton, was that one of the constituents was soluble in the substance of the membrane, while the other was not; this view is not acceptable to physical chemists. Also, the suggestion of Clowes, that the reversal of phase in a membrane of emulsoid structure might explain a changed permeability of membranes, cannot be entertained.

\section{Science and Social Service.}

$T^{H}$

HE presidential address delivered by Sir George $H$. Knibbs at the New Zealand meeting of the Australasian Association for the Advancement of Science in January I923, entitled "Science and its Service to Man," reviews the recent advances in the fields of astronomy, relativity, atomistics, radioactivity, spectroscopy, and various branches of chemistry, including biochemistry, metallurgical chemistry, and a number of technical applications of synthetic chemistry. There is a brief notice of the Rutherford-Bohr theory of atomic structure, and the properties of colloids and of vitamins are discussed, together with the functions of the ductless glands, and their relation to human development. The following extracts from the address are of particular interest:

The highest product of civilisation is not the mere maintenance of man on the planet, but such maintenance as makes him a student of that vast universe of which physically he forms so utterly insignificant a part-a student, developing faculties by means of which he can appreciate beauty, magnificence, majesty, and, indeed, the whole range of things spiritually apperceived or intellectually grasped - a student capable of solving the most apparently hopeless problems.

Nevertheless, in addition to these intellectual gifts, the proper study of science may result in important material advantages. At the same time it must be recognised that scientific advance has introduced previously unsuspected dangers; and while it is essential that nations which desire to preserve their independence should study the application of science to warfare, the terrible weapons which modern discovery places in the hands of unscrupulous nations, and the devastating nature of modern warfare, cause one to tremble for the future history of mankind if means cannot be found to eliminate the evil. Vital statistics clearly show that, with the present normal rate of growth of populations, the world will, in a comparatively short period, become incapable of supporting its teeming millions, in spite of the possibilities of increasing the productivity of the soil.

The overspill of dense populations provokes situations from which apparently there is no escape, for it involves agreement as to expansion, and the much-discussed question of birth control has to be seriously considered. A review of the whole realm of Nature warns us that there may be no way of escaping the great issue. May it not be then accepted that, as long as human nature is what it is now, war is certain, even if it be not inevitable. The way could come in peace, but only through a world-wide discipline, vastly more thoroughgoing than any discipline we dream of at present.

The address concludes with a plea for the creation of a national appreciation of science, for improvement of scientific education, and for the development of research.

"Our hope is to see a new spirit born here. No one knows what lies on the knees of the gods. But there is something within the mind and heart of any great people which responds to the dream of excellence, and inflames when the vision of national destiny is before it. Our Mother-land has had a great past. Is its offspring here in southern seas, illumined by 'the gem pointed cross and the blazing pomp of Orion,' to rise to material, to intellectual, and to moral greatness among earth's peoples? If so, the path is strenuous, but glorious. All visions of ease and luxury are but opiates, and lead to destruction. We shall need to gird ourselves for the task, and create for ourselves a world where our sons, knowing something of the splendid mysteries of the boundless universe, and also of our own little world, will excel in the art of using to the full the heritage our nation has given us. Then indeed will science have rendered noble service to the sons of Australasia." 